\title{
Boden(rechts)ordnungen in Afrika südlich der Sahara. Am Ende der Skandale ein Entwicklungsskandal? ${ }^{1}$
}

\author{
Von Hagen Henry
}

\section{Einführung}

Welchen Sinn könnte es haben, für das angeblich von Krisen gebeutelte Afrika südlich der Sahara (Afrika) ${ }^{2}$ einen zusätzlichen Skandal zu erdenken? Der Sinn ist, daß nur ein radikaler Anstoß bei allen Beteiligten einen Ausweg aus der durch bodenrechtswirksame Maßnahmen mitverursachten Krise ${ }^{3}$ andeuten kann.

Das Auf einandertreffen mehrerer Kulturen in Afrika bewirkt eine einmalige Komplexität auch bei den Bodenordnungen. Der Skandal muß darin bestehen, diese Komplexität zu deuten, damit deren allseits beklagte nachteilige Folgen gewendet werden können, denn das Dilemma Afrikas liegt in der erzwungenen Brache möglicher Synergien unterschiedlicher Kulturen.

Diese Komplexität verböte die Eingrenzung auf die bodenrechtlichen Aspekte der Bodenordnungen, denn diese Eingrenzung ist gerade Teil des Problems. Sie entspricht aber der Vorverbildung des Verfassers. die notwendige Erweiterung ist mit dem Begriff der "bodenrechtswirksamen Maßnahmen" angedeutet.

Nach einer kurzen Darstellung der staatlichen Bodenrechtsmaßnahmen in Afrika wird versucht, deren Scheitern in der Rechtswirklichkeit zu erklären. Als Schlüssel zur Erklärung sollen die Reproduktionsmechanismen von Subsistenzgesellschaften dienen, da in ihnen weiterhin die Mehrheit der Afrikaner lebt, wenn auch nicht immer physisch, so doch

1

Geringfügig geänderte Fassung meines Beitrages zu dem GTZ Sector Project "Relevance of Land Tenure Development for Developing Countries". Die Formulierung der Überschrift ist einem Buchtitel aus den sechziger Jahren ("Le scandale du développement") entlehnt. Er war der Aufruf, Entwicklung als Änderung der Mentalitäten, als Änderung in den Köpfen und nicht als ein Spiel mit meist eingebildeten Zahlen, Maßen und Gewichten zu verstehen.

Das Sprechen über ganz Afrika und die Attribute "afrikanisch" im Gegensatz zu "europäisch" sind gerechtfertigt. Vgl. Henri;, Hagen, Juristische Entwicklungshilfe im Zeichen der Strukturanpassung - Holzweg oder Ausweg aus der Krise?, in: Krisenkontinent Afrika. Ansätze zum Krisenmanagement, Hrsg. Hottes / Gocht / Trappe, Social Strategies, Vol. 27, Basel 1995.

Vgl. die Beiträge in Krisenkontinent Afrika..., a.a.O. (Fn. 2). 
spirituell und rituell. ${ }^{4}$ Es ist dies nur ein Schlüssel aus einem Bund - allerdings ein wenig benutzter.

Am Ende werden einige Gedanken zur Verbesserung der Lage im Rahmen von Projekten und Programmen der Entwicklungszusammenarbeit skizziert, die in den Vorschlag münden, behutsamer zu "intervenieren" ${ }^{5}$ und die dadurch gewonnene Zeit zu nutzen, um Afrika kennenzulernen.

\section{Die staatlichen Bodenrechtsmaßnahmen und ihre Folgen}

\section{Die staatlichen Bodenrechtsmaßnahmen}

Seit Beginn der Kolonialzeit ${ }^{6}$, verstärkt seit der völkerrechtlichen ${ }^{7}$ Konstituierung der afrikanischen Staaten, sind die afrikanischen Bodenordnungen wiederholt Gegenstand staatlicher Gesetzesmaßnahmen gewesen.

Schematisiert dargestellt bestanden diese gesetzlichen Maßnahmen in einer ersten Phase in der Einführung des römisch-rechtlich geprägten Eigentumsbegriffs, der Zuordnung des entsprechenden Rechts an Einzel- oder juristische Personen, der Erfassung von Grund und Boden in Katastern sowie in der Beschränkung des Nachweises von Rechten an Grund und Boden auf Grundbucheintragungen. Seit der völkerrechtlichen Konstituierung der afrikanischen Staaten bestehen diese Maßnahmen - mit unterschiedlichen politischen Begründungen - zusätzlich in eigentumsrechtlicher Zuordnung von Grund und Boden an den Staat,

Nähere Angaben bei Henr̈̈, Juristische Entwicklungshilfe..., a.a.O. (Fn. 2).

5 Niemeijer, David, The Dynamics of African Agricultural History: Is it Time for a New Development Paradigma?, in: Development and Change, 1996, S. 87 ff., schreibt: "(..) development interventions have become one of the major external disruptions" (S. 101). Vgl. auch Berry, S., No Condition is Permanent: The Social Dynamics of Agrarian Change in Sub-Saharan Africa, Madison: The University of Wisconsin Press, 1993.

$6 \mathrm{Da} \beta$ die afrikanischen Bodenordnungen erst in den siebziger Jahren in Kreisen der "land reformers" Interesse weckten (so Platteall, Jean-Philippe, The Evolutionary Theory of Land Rights as Applied to Sub-Saharan Africa: A Critical Assessment, in: Development and Change, 1996, S. 29 m.w.N.), widerspricht dem nicht. Das dürfte vielmehr ein Indiz für eine möglicherweise bestehende Kluft zwischen der "development community" und der Realität sein, soweit nicht die politisch viel mehr beachtete Landreform damit gemeint ist. Im Gegensatz zu Lateinamerika und Asien haben Verteilungsaspekte hinsichtlich Grund und Boden in Afrika jedoch keine nennenswerte Rolle gespielt.

Vgl. zu der hinter dieser Einschränkung stehenden Problematik Henry, Hagen, Political Order in Subsaharan Africa - the European View of African States at Stake, in: Kansainoikeus. Ius Gentium, 1988, S. $131 \mathrm{ff}$. 
der seinerseits unter der Bedingung effektiver Bearbeitung des Bodens gemäß vorgegebener staatlicher Entwicklungspläne (permanente) Nutzungsrechte vergibt. ${ }^{8}$

\section{2. $\quad$ Die Folgen dieser Maßnahmen: Soziale Mißstände und ein nicht beherrschter Rechtspluralismus}

Trotz dieser Gesetzes- und dazugehörender administrativer Reformen und des·Versuchs, das afrikanische Recht verbal und/oder durch gesetzgebenerische Anweisungen in den Stand des Moribunden zu versetzen ${ }^{9}$, lebt das afrikanische Bodenrecht ${ }^{10}$ weiter, wirken die von europäischem Rechtsdenken beeinflußten Gesetzesmaßnahmen nur begrenzt. Der Befund lautet: "Officiellement, la terre appartient à l'Etat... En fait, la terre curcule, et les gens circulent sur les terres, suivant la logique lignagère traditionelle et non pas suivant le droit officiel."

Dieser Befund ist eine zutreffende Momentaufnahme. Er verdeckt aber das durch die Gesetzesmaßnahmen bewirkte Aufbrechen sozialer Strukturen und die durch die potentielle Geltung des sogenannten modernen Rechts entstandene Dynamik: Ein Afrika eigener, noch nicht beherrschter Rechtspluralismus verstärkt die negativen Folgen dieses Aufbrechens.

Gemessen am Ziel der Gesetzesmaßnahmen müssen diese als gescheitert gelten. Erklärtes Ziel war, ein Recht einzuführen, das die für Wirtschaftsreformen, definiert als Wandel von der Subsistenz- zur monetären (Markt)Wirtschaft, notwendigen Investitionen (für Dauerkulturen, Modenmeliorationen, Bewässerung etc.) in der Landwirtschaft und den Zugang

Einzelheiten bei Miinkner, Hans-H., Synthesis of Current State and Trends in Land Tenure, Land Policy and Land Law in Africa, Eschborn: GTZ, 1995; vgl. auch Henr̈̈, Hagen, Die Rolle des Bodenrechts in der ländlichen Entwicklung in Niger, Nigeria und Elfenbeinküste unter entwicklungsrechtlichen Gesichtspunkten, in: Zeitschrift für Rechtsvergleichung, 1982, S. 241 ff.; Henry, Hagen, Das nigerianische Bodenrechtsgesetz 1978, in: Jahrbuch für afrikanisches Recht 1983, S. 45 ff.; Platteau, a.a.O. (Fn. 6), S. 38; CILSS, Hesseling, Gerti / Moussa, Boubakar, Le foncier et la gestion des ressources naturelles au Sahel, Paris: Comité Inter-Etats de lutte contre la sécheresse dans le Sahel, 1994; LeRoy, Étienne, Introduction générale, in: L'appropriation de la terre en Afrique noire, sous la dir. de Émile LeBris, Étienne LeRoy, Paul Mathieu, Paris, Karthala, 1991; Systèmes fonciers à la ville et au villages. Afrique noire francophone, textes réunis et présentés par R. Verdier et A. Rochegude, Paris, Harmattan, 1986, S. 73 ff.

Vgl. Tunc, André, in: Les aspects juridiques du développement économique, dir. par A. Tunc, Paris, Dalloz, 1966, S. 9 ff.

Nicht im Sinne eines vorkolonialen Rechts, sondern in dem oben (Fn. 2) definierten kulturellen Sinne.

11 Desjeux, Dominique, L'Accès à la terre chez les paysans Basundi, in: Enjeux fonciers en Afrique noire, études réunies et présentées par LeBris, LeRoy et Leimdorfer, Paris, ORSTOM et Karthala, 1982, S. $126 \mathrm{ff}$. 
traditionell nicht zugelassener Personen zu Grund und Boden dauerhaft sichern und damit Entwicklung fördern würde. ${ }^{12}$

Die Möglichkeit, durch Grundbucheintragung Eigentum zu begründen oder vom Staat Nutzungskonzessionen zu erhalten, eingetragene Grundstücke als Sicherheit für Kredite oder Waren zu geben oder gar Grundstücke als marktfähige Güter anzusehen, wird fast ausschließlich von exportorientierten Betrieben genutzt, deren Beitrag zur Entwicklung des Landes mit den internationalen terms of trade für die von ihnen hergestellten Waren und der Bereitschaft schwankt, erzielte Gewinne der Volkswirtschaft zur Einfuhr fehlender Nahrungsmittel bereitzustellen. Daneben gibt es städtische Eliten, die das moderne Recht zu Spekulations- oder zu dem Zweck nutzen, ostentative Investitionen in ihren Herkunftsdörfern zu tätigen. Die Zielgruppe der Gesetzesmaßnahmen hingegen kennt die gesetzlichen Möglichkeiten häufig schon mangels Kenntnis der offiziellen Landessprache nicht, hat keinen Zugang zu den Behörden oder sieht keine Notwendigkeit, ihre Bodenrechte durch ein Mittel anerkennen zu lassen, das in ihrem sozialen Umfeld keine Bedeutung hat.

Schlägt die Monetarisierung fehl, weil weder die Bedingungen für die input-Beschaffung noch die Kommerzialisierung der Produkte von den Bauern sichergestellt und/oder von ihnen kontrolliert ist, so sind sie auf die Subsistenzwirtschaft zurückgewiesen, die jedoch den inzwischen eingetretenen Veränderungen wie Steuerpflichten, Schulgebühren oder Verlangen nach Industriegütern nicht entsprechen kann. Nicht selten wächst der durch die Monetarisierung entstehende Kreditbedarf weiter dadurch, daß die Risiken einer wachstumsfördernden Spezialisierung (Dünge- und Schädlingsbekämpfungsmittel etc.) zu decken sind. Die Veränderung materieller Bedürfnisse und die Folgen der in Unkenntnis der damit verbundenen Risiken eingegangenen Kreditverpflichtungen zwingen nicht selten zu persönlicher Lohnarbeit, d.h. zur Abwanderung in die Städte.

So wenig die Bodengesetze zielgemäß effektiv sind, so sehr tragen sie aber dazu bei, traditionelle Sozialsysteme ersatzlos aufzubrechen ${ }^{13}$ und damit das traditionelle Bodenrecht anzuschlagen. Neben Boden ist die menschliche Arbeitskraft weiterhin der wichtigste Produktionsfaktor, ein Abwandern in die Städte trifft daher die afrikanischen Landwirtschaften im Kern. Die häufig zu beobachtende Erstreckung der Gruppensolidarität vom Dorf in die Stadt ist daher begrenzt. In umgekehrter Richtung wirkt diese Solidarität nicht,

Raison, Jean-Pierre, De la prééminence de l'usage du sol à l'émergence d'une question foncière, in: Systèmes fonciers..., a.a.O. (Fn. 8), S. 48.

13

Vgl. Bryde, Brun-Otto, Autochthones und importiertes Recht in Afrika in ihrer Bedeutung für eine "afrikanische Genossenschaft", in: Wege zu einer afrikanischen Genossenschaft, Hrsg. Hans-H. Münkner, Marburg, Institut für Kooperation in Entwicklungsländerm, 1980, S. 129; Gsänger, Hans, The Future of Rural Development, London, Frank Cass, 1994, S. 55; Seidman, Robert, The Fatal Race: Law-Making and the Implementation of Development Goals, in: Third World Legal Studies, 1992, S. 79 ff. 
weil Arbeit in der Stadt häufig eine reine Hoffnung bleibt. Die genannten, eher ostentativen Investitionen städtischer Eliten in ihren Herkunftsdörfern kompensieren den Arbeitskräftemangel nicht. ${ }^{14}$

Dadurch kommt es zu einer gegenseitgen Blockade des traditionellen und des modernen Wirtschaftssektors. ${ }^{15}$ Der moderne Wirtschaftssektor entzieht durch staatlich gestützten Zugang zu als herrenlos deklariertem Land, durch Steuer- und Schulpflicht und durch das Anpreisen materieller Attraktionen über die Massenmedien dem traditionellen Bereich vitale Faktoren. Der traditionelle Sektor widersetzt sich auch deshalb der Ausbreitung des modernen. Die ohnehin geringen Impulse (Nachfrage und Sparvermögen) der Subsistenzgesellschaften für eine monetarisierte Marktwirtschaft werden durch diese Widerstände weiter abgeschwächt. Der moderne Sektor tauscht internationale Unterstützung gegen extrovertiertes Wirtschaftsverhalten ein. Die durch die modernisierungsorientierte Entwicklung eingeleiteten Veränderungen blockieren also eine mögliche Fortentwicklung der traditionellen Landwirtschaft. Diese widersetzt sich der notwendigen Möglichkeit einer zumindest in Teilen nach marktwirtschaftlichen Prinzipien funktionierenden modernen Landwirtschaft und dem damit vorausgesetzten Bodenrecht.

Voraussetzung einer auch die Entwicklungszusammenarbeit leitenden neuen Bodenrechtspolitik ist die Aufhebung dieser Blockade. Sie bedeutet nicht Stillstand. Sie hat vielmehr eine besondere Dynamik. Diese Dynamik spiegelt sich in dem Afrika eigenen Rechtspluralismus. ${ }^{16}$

Die sukzessive transferierten fremden Rechte haben sich nicht mit dem vorhandenen vermischt. Keines der so nebeneinander stehenden Rechte hat sich so durchgesetzt, daß es als allgemein geltendes Recht auf einem Staatsgebiet wirken würde. Der geschichtliche Übergang von einer Phase zur anderen (was meist mit vorkolonial, kolonial und postkolonial beschrieben wird) stellt keine Ablösungszeitpunkte dar, sondern zeigt nur an, wann zu dem schon vorhandenen ein weiteres Recht hinzukam. ${ }^{17}$

Dieser Rechtspluralismus ist ein komplexes, sich ständig veränderndes Gemisch von Rechten unterschiedlicher kultureller Prägung, dessen Elemente von Gruppen wechselnder Zusammensetzung und von Einzelpersonen zu unterschiedlichen Zeiten und gemäß je zum Entwicklungsrecht - ein überfällige Entwicklung, in: Zeitschrift für Rechtsvergleichung, 1985, S. $194 \mathrm{ff}$.

17 Näher Henr̈̈, Hagen, "Afrikanisches" Recht?, in: Zeitschrift für Rechtsvergleichung 1981, S. $242 \mathrm{ff}$. 
anderer Rechtsmaterie eingesetzt wird. Mangels eines seit langem ${ }^{18}$ angemahnten Konfliktrechts und seiner effektiven Durchsetzung ist es anfällig für lex- und forum-shopping durch kulturell Mobile. ${ }^{19}$ Dadurch steigt nicht nur die Zahl der Konflikte ${ }^{20}$, sondern - was schwerer wiegt und die Qualifizierung der Probleme als Krise ${ }^{21}$ rechtfertigt - die Zahl der nicht gelösten, möglicherweise nicht lösbaren Konflikte steigt. Allgemeine Rechtsunsicherheit ist die Folge. Ausweichstrategien wie Verkürzung der Brachen, Überwirtschaftung empfindlicher Böden, Ausweitung der Anbauflächen zeigen in anthropogener Desertifikation und z.T. verheerender Bodendegradation ihre unerträglichen Grenzen. ${ }^{22}$

\section{Die Gründe für das Scheitern der staatlichen Bodenrechtsmaßnahmen}

Allgemein wird weiterhin angenommen, es handele sich bei den Folgen der Gesetzesmaßnahmen um jede beschleunigte Entwicklung notwendigerweise begleitende, behebbare und daher vorübergehende Probleme. Diese allgemeine Annahme beruht auf der in der "Evolutionary Theory of Land Rights" zusammengefaßten Argumentation, wonach bei genügendem Bevölkerungsdruck und Marktintegration (Kommerzialisierung) jede Gesellschaft die europäische Entwicklung zum in Katastern erfaßten und durch Grundbücher nachgewiesenen Individualeigentum an Grund und Boden spontan nachhole ${ }^{23}$ und damit die für die alsbald folgende wirtschaftliche Entwicklung notwendige Rechtssicherheit schaffe. ${ }^{24}$.

Blockieren sich jedoch, wie hier angenommen, die nach unterschiedlichen kulturellen Mustern Handelnden gegenseitig, so handelt es sich nicht um vorübergehende Probleme, sondern um eine Krise, deren Überwindung zunächst eine Analyse der Gründe des Scheiterns der Gesetzesmaßnahmen erfordert. Aus dieser Analyse und nicht aus der Annahme, Afrika werde in jedem Falle die Rechtsentwicklung Europas nachholen ${ }^{25}$, wird sich zeigen, welchen Weg Afrika nach Überwindung der Krise gehen wird.

Kouassigan, Guy-Adjeté, L'Homme et la terre. Droits fonciers coutumiers et droit de propriété en Afrique occidentale, Paris: Berger-Levrault, 1966.

Ausdruck von Stiirzinger, Ulrich, Die Kultur der Mobilität im modemen Afrika, in: GenèveAfrique, 1984, S. 151 ff.; Platteau, a.a.O. (Fn. 6), S. 41, 43 f. m.w.N.; Hesseling, Gerti, Les citadins et le droit à la ville, in: L'appropriation..., a.a.O. (Fn. 8), S. 212.

CILSS, a.a.O. (Fn. 8), S. 1, 5; Mïnkner, a.a.O. (Fn. 8), S. 2.

23 Ausführliche Diskussion dieser Theorie bei Platteau, a.a.O. (Fn. 6), vgl. insbesondere S. 29, 32 m.w.N., 35. 


\section{Die "Evolutionary Theory of Land Rights"}

Die seit langem widerlegte Auffassung, das traditionelle afrikanische Bodenrecht, das die uns gewohnte Rechtssicherheit nicht bietet, sei ungeeignet, wirtschaftliche Veränderungen zu fördern bzw. zu stützen, wird zwar inzwischen auch von den sogenannten Geberorganisationen nicht mehr vertreten. Dem traditionellen afrikanischen Bodenrecht wird Eigendynamik bescheinigt, aber nur insoweit, als es sich "autonom" zu einem System des individuellen Privateigentums verändern werde. ${ }^{26}$ Das Ergebnis der Veränderung gilt also weiterhin als feststehend. Lediglich die Frage, ob und wie dieser angeblich autonome Prozeß gesteuert werden soll, wird anders beantwortet.

Der Einklang der neuen Antwort mit der seit Ende des Ost-West-Konfliktes allgemein hervorgehobenen Bedeutung des - allenfalls staatlich gestützten - Wirkens der civil society ist nicht zu überhören. Die hierin zu Tage tretende nachträgliche Ideologisierung staatlicher Bodenrechtsmaßnahmen in Afrika verdeckt mehrere für die Analyse der aktuellen afrikanischen Krise entscheidende, eng miteinander verwobene Gesichtspunkte, auf die deshalb vorab kurz einzugehen ist.

Zwar ist richtig, daß sich eine erhebliche Zahl unabhängiger afrikanischer Staaten zum Sozialismus als Staatsideologie bekannte. Die oben skizzierten Bodenrechtsgesetzgebungen gab es jedoch auch in nicht sozialistischen Ländern. Die ideologische Überformung war nicht mehr als ein Aspekt eines generellen "suivisme constitutionnel". ${ }^{27}$ In Wirklichkeit ging und geht es um anderes. Diese Rechtspolitiken setzen die während der Kolonialzeit bereits begonnen eeuropäisierung unter dem Stichwort Modernisierung fort. Die Bezeichnung der gesetzlichen Maßnahmen als "Reformen" verschleiert den Sachverhalt. Es ging um den extroi europäischen, nicht um die Reform des vorhandenen afrikanischen Rechts.

Während der Kolonialzeit erzwang die jedem Staat innewohnende Logik Rechtseinheit auf dem gesamten Gebiet unter Einschluß der Kolonien. Die Kolonialmächte duldeten die Fortgeltung des afrikanischen Rechts nur, solange und soweit es ihrem eigenen ordre public nicht entgegenstand. ${ }^{28}$ Seit der völkerrechtlichen Konstituierung der afrikanischen Staaten gilt es, nachträglich die Bedingungen der Entlassung in die staatliche Unabhängigkeit und der Beteiligung an den Entscheidungsprozessen der internationalen Nationalstaatengemeinschaft zu erfüllen. Während Rechtsvereinheitlichung gemeinhin als ein Element nationaler Einheit gilt, wird in bezug auf Afrika häufig übersehen, daß sich die unabhängigen Staaten zunächst erst einmal selbst schaffen müssen. Einheitliches staatliches Bodenrecht ist somit

26

27

28

Platteau, a.a.O. (Fn. 6), S. 30 ff. m.w.N.

Gonidec, Pierre-François, Constitutionnalismes Africains, in: Revue Africaine de Droit International Comparé, 1996, S. $12 \mathrm{ff}$.

Näher Henry, Hagen, Afrikanisches Recht im Banne staatlicher und nationaler Integration und (wirtschaftlicher) Entwicklung, in: VRÜ (1983), S. 293 ff. 
ein Mittel zur Erlangung territorialer Souveränität, einem der drei klassischen Staatselemente. Primäres Ziel der Gesetzesmaßnahmen war und ist daher nicht Wirtschaftsreform, sondern der Versuch, strukturelle Schwächen des Staates zu beheben, Wirtschaftsreform zur Konsolidierung politischer Macht zu betreiben. ${ }^{29}$

Die "Evolutionary Theory of Land Rights" ist in sich nicht schlüssig. Es fällt zunächst auf, daß einmal die Einführung eines grundbuchgesicherten Individualeigentumsrechts an Grund und Boden Kommerzialisierung fördern und dem Bevölkerungswachstum zuvorkommen sollte, während nunmehr Bevölkerungswachstum und Kommerzialisierung das angeblich evolutionär in jeder Gesellschaft angelegte Individualeigentumsrecht an Grund und Boden zur Geltung bringen sollen. Die Theorie belegt nicht, daß Bevölkerungsdruck und Marktintegration immer zu Individualeigentumsrechten an Grund und Boden führen müssen. Das europäische Beispiel ist kein Beleg in einer Welt vieler Kulturen.

Weiterhin ist Bodenknappheit nicht dargelegt. Der aus Bevölkerungszahl und verfügbarer Nutzfläche zu errechnende Quotient ergibt weder weltweit noch auf einzelne Länder bezogen Bodenknappheit. Der Begriff der Knappheit in der "Evolutionary Theory of Land Rights" schließt offensichtlich die Aussage ein, nicht alle Ansprüche auf Zuordnung ein und desselben Grundstückes könnten befriedigt werden. Konflikte sind also nicht in der Knappheit begründet, sondern im Widerspruch solcher Ansprüche.

Darüber hinaus verrechnet der Begriff der Knappheit natürliche Größen (Bevölkerungszahl, Fläche) mit kulturellen Größen (Staats-, Stadt-, Dorf-, Gebiet einer ethnischen Gruppe etc.). Diese in der "Evolutionary Theory of Land Rights" angelegte "Naturalisierung" kultureller Determinanten ist Teil des mit dem Knappheitsbegriff angedeuteten Versuchs, Bodenrecht ausschließlich nach wirtschaftlichen Kriterien zu bestimmen. Dies muß in Afrika schon deshalb zu Fehleinschätzungen führen, da es einen Bodenmarkt, auf dem Knappheit eine Rolle spielen könnte, nicht gibt. Wobei anzumerken wäre, daß die dahinterliegende Auffassung von Wirtschaft nicht kulturübergreifend sein muß.

Um die hier formulierten Bedenken zu stützen, sei gefragt: Wie läßt sich mit der "Evolutionary Theory of Land Rights" das häufig belegte Verhalten der Bauern bei Staudammprojekten erklären? Trotz des Angebots besserer und größerer Ersatzflächen beharren sie auf angestammten Rechten. Wie läßt sich erklären, daß es bei einer Bebauung von nur $2 \%$ der bebaubaren Fläche landlose Bauern gibt? ${ }^{30}$ Wie läßt sich erklären, daß squatter Bewohner (Bevölkerungsdruck) neue Rechte fordern, obwohl der Verbleib in der Stadt mit öko-

Henry, Hagen, Afrikanisches Recht im Banne..., a.a.O. (Fn. 28); Henrj, Political Order..., a.a.O. (Fn. 7); LeRoy', Introduction générale, a.a.O. (Fn. 8), S. 17.

30 LeRoy, Étienne, L'Etat, la réforme et le monopole fonciers, in: L'appropriation..., a.a.O. (Fn. 8), S. 168. 
nomischen Gesichtspunkten kaum begründet werden kann und die squatter-Bewohner sich dessen durchaus bewußt sind?

Schließlich läßt sich mit der "Evolutionary Theory of Land Rights" das Bodenrecht in den Vereinigten Staaten von Amerika und Australien nicht erkären. Zwar wäre es logisch unzulässig, aus der Theorie abzuleiten, daß dort, wo kein Bevölkerungsdruck herrscht, es kein Individualeigentum an Grund und Boden gibt. Gleichwohl sind diese Länder ein Beispiel dafür, daß Bodenrechtsauffassungen losgelöst von dem Verhältnis zwischen Bevölkerungszahl und Nutzfläche reproduziert werden.

Die zweite Bedingung für die in der "Evolutionary Theory of Land Rights" beschriebene Wirkung, die Marktintegration, beruht auf der Theorie induzierter Innovation. ${ }^{31}$ Danach entstehen neue (Rechts)Institute, wenn Veränderungen in der Ausstattung mit Produktionsmitteln und bei anderen Wirtschaftsbedingungen (technische Veränderungen, Preisvariationen etc.) nur deshalb nicht zu höheren Gewinnen führen, weil die alten Institute nicht mehr adäquat sind.

$\mathrm{Zu}$ einer solchen Innovation sei es in Afrika bisher nicht in nennenswertem Umfang gekommen, weil unter den häufig außergewöhnlich schwierigen natürlichen Gegebenheiten technische Innovationen nicht mit dem Tempo des Bevölkerungswachstums Schritt halten könnten. ${ }^{32}$ Darüber hinaus sei das neue Recht bei der Bevölkerungsmehrheit Quelle zusätzlicher Rechtsunsicherheit geworden. ${ }^{33}$ Der Zugang zu Grund und Boden, bisher nur Mitgliedern nach ethnischen Gesichtspunkten geordneter Gruppen möglich, sei nunmehr über staatliche Grundbucheintragung jedem möglich und damit zu einer Frage des Zugangs zu den entsprechenden Behörden geworden. Die bereits genannten Ungleichheiten bei diesem Zugang führten zu einem nicht akzeptierten Vermögenstransfer für Bildungs-, Wirtschafts- und politische Eliten. ${ }^{34}$ Zudem fehle es den Behörden an dem notwendigen Wissen bei der Feststellung sogenannter traditioneller Rechte ${ }^{35}$ sowie an den Voraussetzungen, ein Kataster- und Grundbuchsystem auf dem laufenden zu halten. ${ }^{36}$

Dort, wo es grundbuchgesichertes Individualeigentum an Grund und Boden gibt, sind bisher die vorausgesagten, quasi-automatischen Folgen nicht in nennenswertem Umfang

31

32

33

34

35

36

Platteau, a.a.O (Fn. 6), S. 34, insbesondere Fn. 7 und 8.

Platteau, a.a.O (Fn. 6), S. 73 m.w.N.

Platteau, a.a.O (Fn. 6), S. 39.

Platteau, a.a.O (Fn. 6), S. 45 m.w.N.

Ridell, J.C. / Salacuse, J.W. / Tabachnik, D., The National Land Law of Zaire and Indigenous Land Tenure in Central Bandundu, Zaire, LTC paper no. 92, Madison: University of Wisconsin, 1987, S. $30 \mathrm{f}$

Platteau, a.a.O (Fn. 6), S. 47. 
eingetreten. Es dient kaum als Kreditsicherungsmittel ${ }^{37}$, da die Gerichte vor Zwangsversteigerungen zurückschrecken und sich mangels eines entsprechenden Marktes auch keine Käufer finden würden, da die Kunde von schlechten Erfahrungen mit Land als Kreditsicherungsmittel unter den Bauern die Runde macht oder weil weiterhin andere Sicherungsmittel bevorzugt werden, wie z.B. Verpfändung der (künftigen) Ernte, persönliche Bürgschaften, antizipierte Veräußerung eines Teils der Ernte, Einschaltung von sogenannten informellen Kreditmittlern. ${ }^{38}$

Diese Argumente widerlegen die "Evolutionary Theorie of Land Rights" nicht, wenn es sich bei den genannten Problemen lediglich um Unzulänglichkeiten in der Anwendung und Durchführung des neuen Bodenrechts handelt. Dies wird weiterhin angenommen. Bevölkerungswachstum läßt sich angeblich steuern, "Structural Adjustment Programmes" tragen der Erkenntnis Rechnung, daß die gewollten Ergebnisse nur in einem System auf einander bezogener Maßnahmen zu erzielen sind. Während anfänglich die isolierte Übertragung einzelner Rechtsinstitute genügen sollte, wurden allmählich die Schaffung effizienter Durchführungsbedingungen, schließlich die Übertragung ganzer Systeme im Rahmen von Structural Adjustment Programmes vorgeschlagen. Darin spiegelt sich ein entscheidender Wandel und die Erkenntnis, daß jede Maßnahme der Entwicklungszusammenarbeit auf das Gesamtsystem des sogenannten Empfängers wirkt und daher diese vielfältige Auswirkung berücksichtigt werden muß. Das traditionelle afrikanische Bodenrecht wird also nicht nur von Bodenrechtsreformprojekten betroffen, sondern von einer Vielzahl von Projekten und Programmen, die auf den ersten Blick mit Bodenrecht nichts zu tun haben.

Dies läßt sich aus dem systemischen Charakter von Gesellschaftsstrukturen herleiten. Es läßt sich aber auch daraus ableiten, daß wir entgegen der auch hier bisher zugrunde gelegten Prämisse nicht davon ausgehen können, daß unsere Definition von Bodenrechtsprojekten beim Empfänger als solche wirkt bzw. umgekehrt, daß ein von uns anders definiertes Projekt beim Empfänger nicht die Bodenordnung erschüttert. ${ }^{39}$ So bedeutet die neuere Interpretation des traditionellen Bodenrechts als Sozialversicherungssystem zwar einen wesentlichen Erkenntniszuwachs. Der Schluß, es ließe sich durch Übertragung unseres Sozialversicherungssystems ändern, ist gleichwohl unzulässig. Das traditionelle afrikanische Bodenrecht ist kein Sozialversicherungssystem, sondern es wirkt nur auch als solches.

39 LeRoy, Introduction générale..., a.a.O. (Fn. 8), S. 21 definiert daher den "rapport foncier" als "fait social total". 


\section{Die Unvereinbarkeit der kulturellen Postulate des afrikanischen und des modernen Bodenrechts}

Jenseits an der Übertragbarkeit derartig umfassender Systeme zu hegender Zweifel muß die Frage erlaubt sein, ob nach fast 100 Jahren gleichförmiger Bodenrechtspolitik in Afrika die Defizite $^{40}$ tatsächlich behebbar oder ob nicht die Grundannahmen falsch sind.

Die Schwäche der "Evolutionary Theory of Land Rights" besteht darin, daß sie ohne Begründung auch für Räume außerhalb des Kulturraumes, in dem sie entwickelt wurde, wirtschaftliche von nicht wirtschaftlichen, objektive von subjektiven Faktoren trennt ${ }^{41}$, d.h. die Übertragbarkeit ganzer Systeme ohne Rücksicht auf die Frage postuliert, ob diese Trennungen und Verbindungen universell gültig sind.

Die Annäherung an das Verständnis der durch die Entwicklungszusammenarbeit mitverursachten Blockade stützt sich auf folgende Beobachtung: Offensichtlich gibt es in Afrika eine Sperre gegen Individualeigentum an Grund und Boden. Nachweislich verhindert das traditionelle Bodenrecht Investitionen nicht. Investitionen können auch über lange Zeiträume, sogar über den physischen Tod des Investors hinaus, für diesen gesichert werden. ${ }^{42}$ Das traditionelle Bodenrecht gewährt jedoch dem Einzelnen keine Veräußerungsbefugnis. Nach ethnischen Gesichtspunkten geordnete Gruppen widersetzen sich der Veräußerung von Grund und Boden ${ }^{43}$ an Gruppenfremde. Es besteht also eine exo-intransmissibilite ${ }^{44}$, und Gruppenfremde sind kaum in der Lage, ihre außerhalb der Gruppe gesicherten Eigentumsrechte auszuüben, auch dann nicht, wenn diese Rechte auf staatlich geführten Grundbüchern beruhen. ${ }^{45}$ Nicht anerkanntes Eigentum ist aber kein Eigentum, und: "... en absence du droit de disposer on ne peut, au moins juridiquement, parler de propriété privée" ${ }^{46}$. Der Eigentumsbegriff läßt sich nicht auf weniger als drei Elemente zurückführen, nämlich das Recht des Inhabers über die Nutzungsart und darüber zu bestimmen, wer nutzen darf, sowie die Veräußerungsbefugnis. ${ }^{47}$ Daran zeigt sich auch, daß legal security noch keine security of access bedeuten muß.

40

41

42

43

44

45

46

47

Lucy, William N.R. / Mitchell, Catherine, Replacing Private Property: The Case for Stewardship, in: Cambridge Law Joumal, 1996, S. 570. 
Die Tatsache, daß städtische Eliten, die keinerlei lebensnotwendige Verbindung in ihren Herkunftsdörfern mehr haben, bei denen also das Sozialversicherungsargument nicht zutrifft, dennoch weiterhin meist nur in ihren Herkunftsdörfern in Grund und Boden investieren, bestätigt diese exo-intransmissibilité. Mangelndes Vertrauen in eine Sicherung ihrer Interessen außerhalb der eigenen Gruppe ist lediglich die Kehrseite dieser Tatsache. Es belegt im übrigen, daß bei Fortfall der materiellen Begründungen des Bodenrechts eine überschießende Innentendenz bleibt. Über deren Veränderung lassen sich nur schwer Aussagen machen. Sicher dürfte sein, daß sie sich langsamer verändern als ihre materiellen Grundlagen und daß die Ausblendung subjektiver Elemente aus der "Evolutionary Theory of Land Rights" nicht auf andere Kulturen übertragen werden darf. Jedenfalls ist diese Innentendenz bei der Erklärung der mannigfachen Resistenz gegen das moderne Recht zu berücksichtigen.

Aus der Erklärung und Auslegung der exo-intransmissibilité ergibt sich ein bisher wenig benutzter Schlüssel zum Verständnis der Bodenrechtsprobleme in Afrika als erstem Schritt zu ihrer Lösung. Ausgangspunkt des Einsatzes dieses Schlüssels ist die Überlegung, daß Einzelentscheidungen von Gruppen ihren Sinn aus einem jeder Gruppe inhärenten Reproduktionsmechanismus erhalten.

Nach ethnischen Gesichtspunkten geordnete Subsistenzgruppen (nicht: Subsistenzwirtschaftsgruppen) sind ex definitione geschlossene Gruppen. Die Bereitschaft des Einzelnen, die Gruppe durch Teilnahme und Teilhabe an den gruppenspezifischen Ritualen fortzusetzen, entscheidet über die Mitgliedschaft. ${ }^{48}$ Sie sind wegen ihres Arbeitskräftebedarfs auf Zusammenhalt angewiesen und verhindern daher jeden Emanzipationsversuch einzelner Mitglieder. Die Problematik des in der Emanzipation liegenden Arbeitskraftverlustes für die Gruppe wurde bereits erwähnt. Der Zusammenhang von Arbeitskräften und Bodenordnung in Afrika ist bislang wenig beachtet. ${ }^{49}$ Eine (wirtschaftliche) Differenzierung nach Kriterien, die nicht jeder Mensch qua seines Menschseins (Alters-, Geschlechtergruppen etc.) erfüllen könnte, ist prinzipiell ausgeschlossen, da Gott sich im Menschen und nicht in jedem Menschen einzeln manif estiert.

Nicht das traditionelle Bodenrecht steht Produktivitäts- und Produktionsmengensteigerungen entgegen, sondern das Bedürfnis, den Einzelnen an einer Emanzipation von der Gruppe zu hindern. Waren, die nicht der Subsistenzsicherung dienen, werden deshalb nicht kommerzialisiert, weil dies einer Emanzipation des Herstellers von der Gruppe Vorschub leisten würde. da der Erlös Ersatz für die im Akt der Herstellung gedachte Fortsetzung des Herstellers ist. Eine Überschußproduktion nach Abzug des Subsistenzbedarfs, der den

48

49

Näher Henrï, "Afrikanisches" Recht?, .. (Fn. 17); ählnich Platteau, a.a.O. (Fn. 6), S. 46, insbesondere Fn. 17.

Vgl. die Beiträge in Systèmes fonciers..., a.a.O. (Fn. 8). 
Warenverbrauch zu ostentativen und/oder destruktiven Praktiken einschließt, macht daher keinen Sinn.

Eine allumfassende Zuordnung von Grund und Boden im Sinne eines Eigentumsrechts an einen Menschen findet auch deshalb nicht statt, weil eine Eigentum legitimierende Entäußerung seiner selbst durch Herstellung bei Grund und Boden (im Gegensatz zu den Früchten vgl. oben) nicht gegeben ist.

Da wegen des andernfalls eintretenden Entzugs der Lebensgrundlage nicht allen Mitgliedern der Gruppe die Veräußerung "ihres" Grund und Bodens erlaubt werden kann, besteht ein generelles Veräußerungshindernis. Die Veräußerungsbefugnis, wie sie im modernen Bodenrecht angelegt ist, trifft daher einen Lebensnerv der Subsistenzgruppen.

Mangelnder ${ }^{51}$ wirtschaftlicher Differenzierung entspricht eine mangelnde Ausdifferenzierung politischer Institutionen. Gruppenvorsteher sind keine Machtinhaber, keine ruler, sondern Sprecher der Lebenden, der kommenden Generationen und der Toten, mit denen sie über das Medium Moden kommunizieren. Diese Brücke zu den Stammvätern der Gruppe durch Veräußerung abzubrechen, bedeutete Frevel. ${ }^{52}$ "... en lui [dem Gruppenvorsteher] la parenté se territorialise et la terre se parentélise..." 53

Die Entscheidungsstränge verlaufen horizontal, nicht vertikal. Zwischen den Mitgliedern und der Gruppe vermitteln keine Einheiten, denn die allen Dingen innewohnenden Kräfte, Geister wirken ohne hierarchische Ordnung. ${ }^{54}$ Die Gruppe ist die einzige, mithin keine Instanz, also auch keine Instanz, die sich in eine hierarchische staatliche Struktur eingliedern ließe. Staatlich gesetzte und verwaltete ${ }^{55}$ Bodenrechtsvereinheitlichung kann es nicht geben.

Die mangelnde Verzahnung der nach ethnischen Gesichtspunkten geordneten Gruppen und des Staates verunmöglicht darüber hinaus die Streitbeilegung zwischen Parteien, deren eine

Es sei daran erinnert, daß der Autor diese Erklärung nur für eine weitere mögliche hält, er also die häufigen Infrastrukturdefizite und andere Produktionserschwernisse nicht übersieht.

51 Wertneutral zu verstehen.

52

Verdier, Civilisations paysannes..., a.a.O. (Fn. 44), S. $10 \mathrm{ff}$.

53 Verdier, Civilisations paysannes..., a.a.O. (Fn. 44), S. 21.

54

Vgl. dazu LeRoy, Introduction générale..., a.a.O. (Fn. 8), S. 15.

55 Mathieu, Paul, De la maîtrise de l'eau au contrôle de la terre, in: L'appropriation..., a.a.O. (Fn. 8), S. 6 berichtet sogar über rückläufige Tendenzen, wo zunächst vom Staat verwaltetes Recht allmählich in die anderen Regeln folgende autochthone Verwaltung übergeht. 
einer derartigen Gruppe angehört, während die andere staatlichen Rechtsschutz sucht. ${ }^{56}$ Die staatliche Gerichtsbarkeit ist nicht deshalb wenig effektiv, weil die dort arbeitenden Personen, wie häufig kolportiert, korrupter als andernorts wären, sondern weil sie die Spannungen zwischen den Anforderungen an die Objektivität staatlicher Richter und der Loyalität mit ihrer Gruppe nicht zu lösen vermögen.

Hierarchie und Unterteilung ist ein Strukturelement moderner Staaten, das sich nicht nur durch alle Untergliederungen zieht, sondern auch bei Rechtsinstituten zu finden ist, d.h. die Strukturen der sozialen Institutionen und der sie stützenden Rechtsinstitute sind kongruent und wechselseitig genetisch verknüpft. Dem hierarchisch gegliederten Staat, von dem die mannigfachen Untergliederungen ihre den Staat ständig reproduzierenden Befugnisse ableiten, entspricht das Eigentumsrecht an Grund und Boden, von dem alle anderen sogenannten beschränkt dinglichen Rechte abgeleitet sind und das mehr ist als die Summe dieser Anleitungen. So wenig wie Subsistenzgesellschaften das Übersummenprinzip verkörpern, so wenig können sie Inhaber eines alle anderen Rechte an Grund und Boden zusammenfassenden und über diese hinausreichenden Eigentumsrechtes sein. Die Rechte an Grund und Boden haben in Subsistenzgesellschaften keinen substantiellen, sondern relationellen Wert. ${ }^{57}$ Vor dem Hintergrund unseres hierarchisierten Bodenrechts ist es schwierig, ein Bodenrecht zu verstehen, das aus ebenbürtig konkurrierenden, sich gegenseitig nicht ausschließenden, sondern komplementierenden Nutzungsrechten des Ackerbauers, des Viehhalters, des Jägers, des Nomaden, des Baumfrüchtesammlers, des Medizinmanns u.a. ${ }^{58}$ an ein und demselben Stück Grund und Boden besteht.

Bodenrechte sind also in Afrika eine Funktion der Mitgliedschaft und der Stellung des Einzelnen in einer Gruppe. Die Kriterien der Migliedschaft rücken daher in den Vordergrund $^{59}$ und nicht eine wie immer geartete Rechtfertigung ${ }^{60}$ privaten Individualeigentums an Grund und Boden als ein angeblich notwendiges Element der Personwerdung ohne jeglichen Bezug zu dem menschlichen Bedürfnis, in Gesellschaft zu leben, das möglicherweise Quelle des Rechts überhaupt ist. ${ }^{61}$ Der Versuch, den Ursprung des Eigentums in der

Dem Autor wurde ein Fall in Mali berichtet, wo ein sogenanntes traditionelles Gericht innerhalb weniger Wochen einen bei einem staatlichen Gericht seit mehreren Jahren anhängigen Bodenrechtsstreit löste.

Verdier, Civilisations paysannes..., a.a.O. (Fn. 44), S. 8.

58

59

LeRoy, Introduction générale, a.a.O. (Fn. 8), S. 15.

60

60 Vgl. Hegel, Grundlinien der Philosophie des Rechts, § 46; Locke, J., Two Treatises on Government; Waldron, J., The Right to Private Property, Oxford 1988.

61

Vgl. Li, Tania Murray; Images of Community: Discourse and Strategy in Property Relations, in: Development and Change, 1996, S. 501 f. m.w.N.; Verdier, Civilisations paysannes..., a.a.O. (Fn. 44), S. 6. Zu diesem möglichen Ursprung des Rechts vgl. Maridakis, George S., Droit, droit mon- 
Ersitzung, d.h. im Zeitablauf zu sehen ${ }^{62}$, ist eine kulturspezifische Verkürzung des Zeitbegriffs, die es ermöglicht, dem Zeitablauf rechtsverändernde Wirkung beizumessen und damit der in der Zeit begrenzten Einzelperson Rechte zuzuordnen. Steht aber die soziale Zeit der Gruppe im Vordergrund, die mehr ist als Summe der Lebensdauer der einzelnen Mitglieder, so kann Zeit weder Rechtsänderungen bewirken, noch können dem vergänglichen Mitglied Rechte unabhängig von der Gruppe eingeräumt werden. 63 "... il y a d'un côté les droits du groupe en tant que ses membres le constituent; de l'autre, il y a des individus qui tiennent leurs droits de leur appartenance au groupe." ${ }^{64}$ Genauer wäre die Gruppe als Individuum zu bezeichnen. Die Konfusion von Person und Individuum im westlichen Recht ist kulturspezifisch. ${ }^{65}$

In diesem Zusammenhang steht auch die unterschiedliche Konzeption von Grund und Boden. Bei uns zweidimensionale, in Grundstücke zerlegbare Fläche, ist er bei den meisten Subsistenzgesellschaften Afrikas ein espace non fragmenté und kann schon deshalb nicht zur Handelsware werden. ${ }^{66}$ Bohannan schloß aus dem Vorrang der Gruppenzugehörigkeit und den Unterschieden in der Wahrnehmung der Dimensionen auf das Fehlen einer geographical map in diesen Gesellschaften. Wir hingegen sind es nicht gewohnt, im Zusammenhang mit Bodenrecht social maps zu lesen. ${ }^{67}$

\section{Schluß}

Ist das moderne Bodenrecht nicht nur wenig effektiv, sondern in sozialer und wirtschaftlicher Hinsicht sogar schädlich, weil die Mehrzahl der Bevölkerungen in Gruppenverbänden lebt, deren kulturelle Codierung nicht mit der des neuen Rechts übereinstimmt, so stellt sich die Frage, ob nicht einer gruppenzentrierten (community based) Entwicklung der

dial, droit comparé, in: Problèmes contemporains de droit comparé, T. 2, Tokio: Institut japonais de droit comparé, 1962, S. 193 ff.

So Michelet, Elisabeth, La règle du non-cumul du possessoire et du pétitoire, Paris: Pichon et Durand-Auszias, 1973, S. 7.

Näher Henr̈̈, "Afrikanisches" Recht?, a.a.O. (Fn. 17).

Verdier, Raymond, Féodalités et collectivismes africains, in: Présence Africaine, 1961, S. 99; Gonidec, a.a.O. (Fn. 27), S. 15.

$\mathrm{Zu}$ den unterschiedlichen Konzeptionen von Person vgl. Nachweise bei Verdier, Civilisations paysannes..., a.a.O. (Fn. 44), S. 8, Fn. 4.

Vgl. Plattealu, a.a.O. (Fn. 6), S. 50 m.w.N.; LeRoy, Introduction générale, a.a.O. (Fn. 8), S. 15.

Bohannan, Paul, Land, Tenure, Land Tenure, in: African Agrarian Systems, Oxford University Press, 1963. 
Vorrang zu geben sei. Gestützt auf Vorschläge des Land Tenure Centers ${ }^{68}$ scheint u.a. die Weltbank inzwischen diesen Weg zu gehen.

Die Bedenken dagegen überwiegen:

a) Die Begründungen für den community approach decken sich mit Argumenten gegen die von Hardin aus einer angeblichen "tragedy of the commons" 70 abgeleiteten Notwendigkeit privaten Eigentums an allen Gütern. Es wird bezweifelt, daß "commons" das Bodenrecht afrikanischer Subsistenzgruppen beschreibt. ${ }^{71}$

b) Bei unveränderten völkerrechtlichen Entscheidungsstrukturen muß eine derartige Politik auf den Widerstand der afrikanischen Staaten stoßen. Die oben genannten Gründe für die bisherigen Bodenrechtspolitiken erklären diesen Widerstand, denn "the distinguishing characteristic of common property management systems is that their primary legitimacy is drawn from the community in which they operate and not from the nation state in which they are located" 72 .

Hinzu kommt die Entwicklung neuer globaler, jedes Land, jeden Bürger betreffender, von den Staaten nicht kontrollierter und/oder kontrollierbarer Entscheidungsstrukturen, nämlich globale Märktẹ, weltweit operierende Oligopole der Wissensproduktion und der Wissens- und Informationsverbreitung. Während die afrikanischen Staaten in der Diskussion um eine neue Weltwirtschaftsordnung hoffen konnten, ihrer formellen Gleichheit in den Entscheidungsprozessen der internationalen Staatengemeinschaft durch Verbesserung der wirtschaftlichen Lage tatsächliches Gewicht zu verleihen, haben sie in den neben dieser Gemeinschaft entstandenen, dieser zumindest ebenbürtigen neuen Entscheidungsstrukturen keinerlei Gewicht. "Weniger Staat", "mehr civil society", "community approach" ... sind daher nur in sich schlüssige Appelle. ${ }^{73}$ Der Ausschluß von diesen neuen Entscheidungsstrukturen läßt auch die Forderung nach Endogenisierung der Entwicklungszusammenarbeit für wenig realisierbar erscheinen.

Bruce, J.W., Do Indigenous Tenure Systems Constrain Agricultural Development?, in: Land in African Agrarian Systems, ed. by Basset and Crummey, Madison: The University of Wisconsin Press, 1993, S. $50 \mathrm{f}$.

73 Ki-Zerbo, a.a.O. (Fn. 14), S. 103 spricht von einer "unopposed strategic role of the state" in diesem Zusammenhang. 
c) Die Gefahr, daß sich neue "communities" allein zum Zwecke internationaler Förderung konstituieren, ist genauso real wie die Verbreitung von NGOs zu diesem Zweck. ${ }^{74}$ Um dieser Gefahr entgegenzuwirken, müßte die Entwicklungszusammenarbeit jedenfalls stärker als bisher vom Wissen der Ethnologen und Anthropologen Gebrauch machen.

d) Ein Konzentrieren auf bestehende Gruppenverbände übersähe schließlich, daß diese sich auflösen ${ }^{75}$ und ginge damit erneut an der afrikanischen Wirklichkeit vorbei.

Trotz der Beschreibung des Bodenrechtszustandes in Afrika als Blockade darf nicht übersehen werden, daß der Rechtspluralismus kein Gleichgewichtszustand ist. Die innerstaatlichen, die internationalen und vor allem global wirkende Kräfte fördern die Individualisierung des einzelnen Menschen, seine Emanzipation von der Gruppe, die privative Aneignung von Grund und Boden ${ }^{76}$ (im Gegensatz zu einer "affectation à un usage" ${ }^{\text {"77 }}$ ).

Was die innerstaatlichen Kräfte angeht, so sei an den bereits skizzierten fortgesetzten Zwang erinnert, die Elemente moderner Staatlichkeit zu sichern. Hinzu kommt, daß staatliche Macht in den Städten ihre wirtschaftliche Basis findet ${ }^{78}$ und die Bodenrechtsprobleme dort vorrangig gelöst werden müssen. ${ }^{79}$

Entwicklungszusammenarbeit ist interstaatliche Zusammenarbeit. Damit ist das Modell festgelegt. Im übrigen kann auch Entwicklungszusammenarbeit nicht den weltweiten Trend zur Übernahme modernen europäischen Rechts aufhalten. Mehr noch als globale Märkte einheitliches Recht voraussetzen, schaffen sie Mechanismen einer Angleichung an das Recht des stärkeren Marktteilnehmers. ${ }^{80}$ Unter dem Gesichtspunkt der Verdrängung kulturell anders codierten Rechts liegt darin ein Verstoß gegen das Menschenrecht auf Entwicklung.

Vgl. $L i$, a.a.O. (Fn. 61); Bertrand, Alain, Les fonciers forestiers, in: L'appropriation..., a.a.O. (Fn. 8), spricht davon, daß collectivités locales "geschaffen" werden müßten. Ki-Zerbo, a.a.O. (Fn. 14), S. 105, $111 \mathrm{f}$.

Vgl. L'appropriation..., a.a.O. (Fn. 8), S. 158; Banque Mondiale, L'Afrique subsaharienne de la crise à une croissance durable, Étude de prospective à long terme, Washington, 1989. "Private property is the gratest interest in a thing admitted by a mature legal system" (Honoré, A.M., Making Law Bind, Oxford, 1987, S. 161). Ersetzt man "mature" durch "modem" oder "zivilisiert", so ergibt sich eine bemerkenswerte Mischung aus fortgesetzter mission civilisatrice und einer Mystifizierung eines auch bei uns nicht mehr so umfassenden Eigentumsbegriffs (vgl. Luc)' Mitchell, a.a.O. [Fn. 47], S. 570).

LeRoy, Introduction générale..., a.a.O. (Fn. 8), S. 11.

Ki-Zerbo, a.a.O. (Fn. 14), S. 105 spricht von einem "bias towards the national centre". 2). 
Bestätigt diese Entwicklung nicht doch die Richtigkeit der "Evolutionary Theory of Land Rights"? Vieles deutet darauf hin. Eine Zwangsläufigkeit sozialer Prozesse gibt es jedoch nicht. $^{81}$ Der Entwicklungsskandal, der die Annahme einer solchen Zwangsläufigkeit stoppte, könnte darin bestehen, uns in der Entwicklungszusammenarbeit auf das zu beschränken, was wir realistischerweise beherrschen können. Dazu gehört:

- Feststellen, daß fast jedes Projekt oder Programm der Entwicklungszusammenarbeit auf die Bodenordnung wirkt, jedenfalls im Umweg über seinen Einfluß auf kulturelle Postulate $^{82}$, sowie: danach handeln!

- Bodenordnung auf ein anthropologisch (d.h. bar kulturspezifischer Festlegungen) begründbares Grundmodell zurückführen ${ }^{83}$ und dieses Grundmodell mit den zumindest in der überschießenden Innentendenz noch sichtbaren Normen und Prinzipien aller beteiligten Rechtsordnungen füllen ${ }^{84}$ und dabei auch nichtstaatliches Recht als Recht anerkennen.

- Überprüfen, ob die eigenen politischen Gegebenheiten diesen anthropologischen Möglichkeiten den notwendigen Freiraum bieten.

- Die kulturell unterschiedlich codierten Rechtsordnungen so miteinander verzahnen, daß die negativen Energien der kulturell Mobilen umgekehrt werden und daß métissages möglich bleiben ${ }^{85}$, d.h. Verzahnung im Sinne einer articulation, nicht im Sinne einer Integration und nicht nur im Sinne von Dezentralisierung und Dekonzentration. ${ }^{86}$

- Einsehen, daß die unser Handeln provizierende Rechtsunsicherheit in Afrika bleiben wird, solange nach ethnischen Gesichtspunkten geordnete Gruppen fortbestehen, greifbar oder auch nur in dem, was hier mit überschießender Innentendenz bezeichnet wurde. Diese Rechtsunsicherheit beruht zu einem geringeren Teil darauf, daß die äuße-

Vgl. Rochegude, A.., De la nécessité d'un droit des terres, in: Systèmes fonciers..., a.a.O. (Fn. 8), S. 59. Bohannan, Land..., a.a.O. (Fn. 67), S. 106, und Verdier, Civilisations paysannes..., a.a.O. (Fn. 44), S. 5 schlagen ein solches Modell vor.

85 Ähnlich LeRoy', L'Etat..., a.a.O. (Fn. 30), S. 179; Ki-Zerbo, a.a.O. (Fn. 14), S. 106.

LeRoy, Étienne, Associer l'usager à la gestion foncière paritaire et décentralisée, in: L'appropriation..., a.a.O. (Fn. 8), S. 334. matrice culturelle africaine et européenne". Vgl. auch Henrj;, Hagen, Co-operative Law and Human Rights, in: The relationship between the state and cooperatives in co-operative legislation, ed. ILO, Genève: ILO 1994, S. 35 f. 
ren Zeichen der Sicherheit - Kataster und Grundbuch - sowie deren Realisierung im Konfliktfall fehlen. Zum weitaus größeren Teil beruht sie darauf, daß wir Afrika nicht verstehen $^{87}$, daß unser Wissen über Afrika unser Wissen ist. ${ }^{88}$ "Le rapport foncier est surtout en Afrique traditionnelle - une relation 'imaginée' entre les hommes à propos de l'espace et qui n'existe que selon des conventions qui s'inscrivent dans les systèmes d'idées avant d'être matérialisées dans l'espace social" ${ }^{89}$ Die methodologischen Konsequenzen aus dieser Feststellung sind erheblich und dürften nicht von allen akzeptiert werden.

Vgl. zu dieser Problematik Preiswerk, Roy, Kognitive Grundlagen westlichen Handelns in der Dritten Welt, in: Entwicklungsprobleme interdisziplinär, Hrsg. Peter / Hauser, Bem u.a.: Haupt, 1976, S. 153 ff.

89 LeRoy, Introduction générale..., a.a.O. (Fn. 8), S. 12. 


\section{The Structure of Land Tenure Legislation in Africa South of the Sahara}

\section{By Hagen Henrÿ}

The author points to the fact that a far greater number of development projects and programmes than those directly addressing land issues do influence land law. Land law reforms have contributed to social and economic problems and have led to a unique legal pluralism. This is blocking the development of communities living according to culturally different laws. The author discusses, and finally rejects, the Evolutionary Theory of Land Rights (ETLR) and the community approach to land law reforms by international donors. Land shortage and market integration as a consequence of induced technological innovations, the two conditions that, according to the ETLR, automatically lead to individually, registered ownership of land, are pure assumptions. The community approach risks to detect communities where there are none, and States will resist, because they must maintain sovereignty over land.

Understanding the cultural difference between the land law as lived by the majority of the people and the land law introduced through land law reforms might be a first step towards unblocking the situation. The organizational principles of subsistence societies in which the majority of Africans continue to live, either physically or spiritually, are one key to that understanding. The next step might be to link the different systems in order to use the synergetic effects. 